\title{
Random collision model for interacting populations of two species and the fluctuation-dissipation theorem - the law of large numbers and the central limit theorem
}

\author{
Yasunori OKABE, Hajime MANO and Yoshiaki ITOH \\ (Received January 13, 1994)
}

\section{$\S 1$. Introduction}

Problems of interspecific competitions have been studied by many authors since LOTKA [9] and VOLTERRA [19]. EHRENFEST's urn model was discussed by KAC [5] and MORAN [10] studied an urn model for the random genetic drift. ITOH $[2,3,4]$ introduced a random collision model which is an urn model for competing species in finite numbers of individuals of several types interacting with each other and studied the problem of coexistence of species.

KogAn, LiptSER, SHIRYAEV and SMORODINSKy $[7,8]$ investigated a queuing model which is formulated in the framework of semimartingales. By using shochastic calculus, they derived an ordinary differential equation from the weak law of large numbers and a Gaussian diffusion process from the central limit theorem for the model.

In this paper we shall discuss the random collision model of two pecies in $[2,3,4]$ which is described by a random time change of a Poisson process. We shall in $\S 2$ show the existence and uniqueness of solutions of the random collision model stated the above. By proving that the random time is a stopping time, we shall in $\S 3$ show that the stochastic process, the unique solution of our model, is a Markov process and has a stochastic structure to be called the fluctuation-dissipation theorem in statistical physics (KuBO [6] and OKABE [11-16]), which is assumed in the onedimensional queuing model in $[7,8]$. Therefore the weak law of large numbers for our model can be proved by the same method as in $[7,8]$. The purpose in $\S 4$ is to prove the strong law of large numbers for our random collision model. $\S 5$ treats a central limit theorem for our model by the same method as in $[7,8]$.

In the future, we shall give a systematic treatment of some random collision models such as paper-scissors-stone model and Ohta-Kimura model for interacting populations and characterize certain qualitative 
property of the fluctuation-dissipation theorem for random collision models. Parts of our project have been already appeared in [17, 18].

The authors would like to thank Professor T. SHIGA for pointing a simplified proof of Step 4 in the proof of THEOREM 4.2 and his other valuable advices.

\section{$\S 2$. A random collision model of two species}

Let us consider a population of two types of individuals in which individuals interact randomly with each other. Changes occur by interactions only between particles of different types. If two individuals of different types interact, then two individuals of the dominant type result from the interaction. Hence the total number of the particles is invariant under interactions.

We set any fixed positive integer $M$ which denotes the total number of the particles. For each $j \in\{1,2\}$, let $X_{j}^{(M)}(*)$ be a stochastic process which denotes the number of individual of type $j$. We assume that the type 1 is dominant than the type 2 and that each of the individuals is described by the time change of a standard Poisson process $N(*)$ defined on a probability space $(\Omega, \mathscr{B}, P)$ in a stochastic integral form as

$$
\left\{\begin{array}{l}
X_{1}^{(M)}(t)=X_{1}^{(M)}(0)+N\left(\frac{\lambda}{M} \int_{0}^{t} X_{1}^{(M)}(s) X_{2}^{(M)}(s) d s\right) \\
X_{2}^{(M)}(t)=X_{2}^{(M)}(0)-N\left(\frac{\lambda}{M} \int_{0}^{t} X_{1}^{(M)}(s) X_{2}^{(M)}(s) d s\right) \\
X_{1}^{(M)}(0)+X_{2}^{(M)}(0)=M
\end{array}\right.
$$

where $\lambda$ is a positive number and $X_{j}^{(M)}(0)$ are initial values of $X_{j}^{(M)}(*)(j=$ $1,2)$, which are defined on the probability space $(\Omega, \mathscr{B}, P)$.

Now we shall prove the existence and uniqueness of solutions of equation (2.1). We denote by $\left\{\tau_{i} ; i=0,1,2, \cdots\right\}$ the set of jumping times of the standard Poisson process $N(*)$.

THEOREM 2.1. There exists a unique solution of equation (2.1) and it is represented in the following form

$$
\begin{aligned}
& X_{1}^{(M)}(t)=X_{1}^{(M)}(0)-1+\sum_{i=0}^{M-X^{(M)}(0)} \chi_{\left[\sigma_{i}^{(M)}, \infty\right)}(t), \\
& X_{2}^{(M)}(t)=X_{2}^{(M)}(0)+1-\sum_{i=0}^{M-X_{X}^{(M)}(0)} \chi_{\left[\sigma_{i}^{(M)}, \infty\right)}(t),
\end{aligned}
$$

where $\sigma_{k}^{(M)}(0 \leq k \leq M)$ are given by 


$$
\left\{\begin{array}{rrr}
\sigma_{0}^{(M)}=0, & \text { for } 1 \leq k \leq M, X_{1}^{(M)}(0)=0 \text { or } M, \\
\sigma_{k}^{(M)}=\infty & \tau_{i}-\tau_{i-1} \\
\sigma_{k}^{(M)}=\sum_{i=1}^{k} \frac{\left(X_{1}^{(M)}(0)+i-1\right)\left(1-\left(X_{1}^{(M)}(0)+i-1\right) / M\right)}{\lambda} & \text { for } 1 \leq k \leq M-X_{1}^{(M)}(0), X_{1}^{(M)}(0) \neq 0, M, \\
\sigma_{k}^{(M)}=\infty & \text { for } M-X_{1}^{(M)}(0)<k \leq M, X_{1}^{(M)}(0) \neq 0, M .
\end{array}\right.
$$

PROOF. Firstly we shall prove that the solution of equation (2.1) is unique. Let $X_{j}^{(M)}(*)(j=1,2)$ be a solution of equation (2.1). For each fixed $t \in[0, \infty)$, we define

$$
T^{(M)}(t) \equiv \frac{\lambda}{M} \int_{0}^{t} X_{1}^{(M)}(s) X_{2}^{(M)}(s) d s .
$$

Every time when the function $T^{(M)}(*)$ comes to the jumping times of the standard Poisson process $N(*)$, the stochastic process $X_{1}^{(M)}(*)$ increases in the width of one. We define $\sigma_{k}^{(M)}$ by

$$
\sigma_{k}^{(M)} \equiv \inf \left\{t \geq 0 ; T^{(M)}(t)=\tau_{k}\right\} \quad(1 \leq k \leq M) .
$$

It is to be noted that $\sigma_{0}^{(M)}=0$.

When $X_{1}^{(M)}(0)=0$ or $M$, we can see that, for any $t>0, T^{(M)}(t)=0$ and so $X_{1}^{(M)}(t)=X_{1}^{(M)}(0)$. Hence it follows that (2.2), (2.3) and (2.4) hold.

When $X_{1}^{(M)}(0) \neq 0, M$, if $\sigma_{k-1}^{(M)} \leq t<\sigma_{k}^{(M)}$ for $1 \leq k \leq M-X_{1}^{(M)}(0)$, and so $\tau_{k-1} \leq T^{(M)}(t)<\tau_{k}$, then

$$
X_{1}^{(M)}(t)=X_{1}^{(M)}(0)+N\left(T^{(M)}(t)\right)=X_{1}^{(M)}(0)+k-1 .
$$

Therefore

$$
\begin{aligned}
X_{1}^{(M)}(t) & =X_{1}^{(M)}(0)-1+\sum_{i=0}^{k-1} 1 \\
& =X_{1}^{(M)}(0)-1+\sum_{i=0}^{M-X_{1}^{(M)}(0)} \chi_{\left[\sigma_{i}^{(M)}, \infty\right)}(t) .
\end{aligned}
$$

If $t \geq \sigma_{M-X_{1}^{(M)}(0)}^{(M)}$, then $T^{(M)}(t)=\tau_{M-X_{1}^{(M)}(0)}^{(M)}$ and so

$$
\begin{aligned}
X_{1}^{(M)}(t) & =X_{1}^{(M)}(0)+N\left(T^{(M)}(t)\right)=X_{1}^{(M)}(0)+\left(M-X_{1}^{(M)}(0)\right) \\
& =X_{1}^{(M)}(0)-1+\sum_{i=0}^{M-X_{1}^{(M)}(0)} 1 \\
& =X_{1}^{(M)}(0)-1+\sum_{i=0}^{M-X_{1}^{(M)}(0)} \chi_{\left[\sigma_{i}^{(M)}, \infty\right)}(t) .
\end{aligned}
$$

Thus we see that (2.2) and (2.3) hold. Furthermore it can be found from (2.6) that the random times $\sigma_{k}^{(M)}$ satisfy a recursive relation (2.4), 
which concludes that the uniqueness of solutions of equation (2.1) holds.

Conversely, let $X_{1}^{(M)}(*)$ be the stochastic process defined by $(2.2)$ and put $X_{2}^{(M)}(*)=M-X_{1}^{(M)}(*)$. It is easy to see that $X_{j}^{(M)}(*)$ are right continuous and have the left-hand side limit $(j=1,2)$.

When the time $t$ is involved in the interval $\left[\sigma_{k-1}^{(M)}, \sigma_{k}^{(M)}\right)(1 \leq k \leq$ $\left.M-X_{1}^{(M)}(0)\right)$, we have

$$
\begin{aligned}
T^{(M)}(t)= & \frac{\lambda}{M} \sum_{i=1}^{k-1} \int_{\sigma_{i=1}^{(M)}}^{\sigma_{i}^{(M)}} X_{1}^{(M)}(s) X_{2}^{(M)}(s) d s+\frac{\lambda}{M} \int_{\sigma_{k-1}^{(M)}}^{t} X_{1}^{(M)}(s) X_{2}^{(M)}(s) d s \\
= & \sum_{i=1}^{k-1} \frac{\lambda}{M}\left(X_{1}^{(M)}(0)+i-1\right)\left(M-X_{1}^{(M)}(0)-(i-1)\right)\left(\sigma_{i}^{(M)}-\sigma_{i-1}^{(M)}\right)+ \\
& +\frac{\lambda}{M}\left(X_{1}^{(M)}(0)+k-1\right)\left(M-X_{1}^{(M)}(0)-(k-1)\right)\left(t-\sigma_{k-1}^{(M)}\right) \\
= & \tau_{k-1}+\frac{\lambda}{M}\left(X_{1}^{(M)}(0)+k-1\right)\left(M-X_{1}^{(M)}(0)-(k-1)\right)\left(t-\sigma_{k-1}^{(M)}\right) .
\end{aligned}
$$

Therefore it follows that $\tau_{k-1} \leq T^{(M)}(t)<\tau_{k}$ and so $N\left(T^{(M)}(t)\right)=k-1$.

On the other hand, we see from (2.2) that when the time $t$ is involved in the interval $\left[\sigma_{k-1}^{(M)}, \sigma_{k}^{(M)}\right), X_{1}^{(M)}(t)=X_{1}^{(M)}(0)-1+k$ and so $X_{1}^{(M)}(t)=X_{1}^{(M)}(0)$ $+N\left(T^{(M)}(t)\right)$.

When $t \geq \sigma_{M-X^{(m)}(0)}^{(M)}$, we find that

$$
\begin{aligned}
T^{(M)}(t) & =\frac{\lambda}{M} \sum_{i=1}^{M-X_{1}^{(M)}(0)} \int_{\sigma_{i=1}^{(M)}}^{\sigma_{i}^{(M)}} X_{1}^{(M)}(s) X_{2}^{(M)}(s) d s+\frac{\lambda}{M} \int_{\sigma_{M}^{(M)}-x_{i}^{(M)}\left({ }_{(0)}\right.}^{t} X_{1}^{(M)}(s) X_{2}^{(M)}(s) d s \\
& =\sum_{i=1}^{M-X_{1}^{(M)}} \frac{\lambda}{M}\left(X_{1}^{(M)}(0)+i-1\right)\left(M-X_{1}^{(M)}(0)-(i-1)\right)\left(\sigma_{i}^{(M)}-\sigma_{i-1}^{(M)}\right) \\
& =\tau_{M-X_{1}^{(M)}(0) .}
\end{aligned}
$$

Hence it follows that $X_{1}^{(M)}(t)=X_{1}^{(M)}(0)+\left(M-X_{1}^{(M)}(0)\right)=X_{1}^{(M)}(0)+N\left(T^{(M)}(t)\right)$.

Consequently we find that the stochastic process $X_{j}^{(M)}(*)(j=1,2)$ satisfies equation (2.1).

Q.E. D.

\section{$\S 3 . \quad$ A stochastic structure for the model : the fluctuation-dissipation theorem}

We define a reference family $\left(\mathscr{F}_{t}^{(M)} ; t \geq 0\right)$ by

$$
\mathscr{F}_{t}^{(M)} \equiv \sigma\left(X_{1}^{(M)}(0), N(s) ; 0 \leq s \leq t\right) .
$$

Let $X_{j}^{(M)}(*)(j=1,2)$ be the unique solution of equation (2.1). Similarly as in (2.5), we define for each $t \in[0, \infty)$ a random time $T^{(M)}(t)$ by 


$$
T^{(M)}(t) \equiv \frac{\lambda}{M} \int_{0}^{t} X_{1}^{(M)}(s) X_{2}^{(M)}(s) d s .
$$

We shall prove the fundamental lemma to investigate certain stochastic structure for our random collision model (2.1).

LEMMA 3.1. For each $t \in[0, \infty)$, the random time $T^{(M)}(t)$ is a stopping time with respect to the reference family $\left(\mathscr{F}_{t}^{(M)} ; t \geq 0\right)$.

PROOF. We have to prove that, for any $t, u \in[0, \infty)$,

$$
\left(T^{(M)}(t) \leq u\right) \equiv\left\{\omega \in \Omega, T^{(M)}(t)(\omega) \leq u\right\} \in \mathscr{F}_{u}^{(M)} .
$$

Since the function $T^{(M)}(*)$ is strictly increasing and continuous in $\left[0, \sigma_{M-X_{i}^{(M)}(0)}\right)$, we note that, for any $u, \tau_{k} \leq u<\tau_{k+1} \leq \tau_{M-X_{i}^{(M)}(0)}$, there exists an inverse value $T^{(M)-1}(u)$ such that

$$
\begin{aligned}
T^{(M)-1}(u)=\sum_{i=1}^{k} & \frac{\tau_{i}-\tau_{i-1}}{\lambda\left(X_{1}^{(M)}(0)+i-1\right)\left(1-\left(X_{1}^{(M)}(0)+i-1\right) / M\right)}+ \\
& +\frac{u-\tau_{k}}{\lambda\left(X_{1}^{(M)}(0)+k-1\right)\left(1-\left(X_{1}^{(M)}(0)+k-1\right) / M\right)} .
\end{aligned}
$$

Now we decompose the measurable event $\left(T^{(M)}(t) \leq u\right)$ into

$$
\begin{aligned}
& \left(T^{(M)}(t) \leq u\right) \\
& =\left(X_{1}^{(M)}(0)=0, T^{(M)}(t) \leq u\right) \cup\left(X_{1}^{(M)}(0)=M, T^{(M)}(t) \leq u\right) \cup\left[\bigcup _ { l = 1 } ^ { M - 1 } \left\{\left(X_{1}^{(M)}(0)=l\right) \cap\right.\right. \\
& \left.\left.\quad \cap\left\{\left(\tau_{M-l} \leq u, T^{(M)}(t) \leq u\right) \cup\left\{\bigcup_{k=0}^{M-l-1}\left(\tau_{k} \leq u<\tau_{k+1}, T^{(M)}(t) \leq u\right)\right\}\right\}\right\}\right] \\
& =\left(X_{1}^{(M)}(0)=0\right) \cup\left(X_{1}^{(M)}(0)=M\right) \cup\left\{\bigcup_{l=1}^{M-1}\left(A_{l} \cup B_{l}\right)\right\},
\end{aligned}
$$

where, for $l, 1 \leq l \leq M-1$,

$$
\left\{\begin{array}{l}
A_{l} \equiv\left(X_{1}^{(M)}(0)=l\right) \cap\left\{\bigcup_{k=0}^{M-l-1}\left(\tau_{k} \leq u<\tau_{k+1}, t \leq T^{(M)-1}(u)\right)\right\}, \\
B_{l} \equiv\left(X_{1}^{(M)}(0)=l\right) \cap\left(\tau_{M-l} \leq u, T^{(M)}(t) \leq u\right) .
\end{array}\right.
$$

Here we used the fact that if $X_{1}^{(M)}(0)=0$ or $M$, then $T^{(M)}(t)=0$ for any $t \in[0, \infty)$.

We fix any $l, 1 \leq l \leq M-1$. Since it then follows from (3.4) that, for any $k, 0 \leq k \leq M-l$,

$$
\left(t \leq T^{(M)-1}(u)\right) \in \mathscr{F}_{\tau_{k}}^{(M)} \equiv\left\{C \in \mathscr{B} ; C \cap\left(\tau_{k} \leq v\right) \in \mathscr{F}_{v}^{(M)} \text { for any } v \in[0, \infty)\right\},
$$

we see that $\left(\tau_{k} \leq u, t \leq T^{(M)-1}(u)\right) \in \mathscr{F}_{u}^{(M)}$. Moreover, since $\left(u<\tau_{k}\right) \in \mathscr{F}_{u}^{(M)}$, we find that $A_{l} \in \mathscr{F}_{u}^{(M)}$.

In addition to this, since if $X_{1}^{(M)}(0) \neq 0, M$, then the function $T^{(M)}(*)$ 
has the maximal value $\tau_{M-X_{i}^{(m)}(0) \text {, we see that }}$

$$
\left(X_{1}^{(M)}(0)=l\right) \cap\left(\tau_{M-l} \leq u, T^{(M)}(t) \leq u\right)=\left(X_{1}^{(M)}(0)=l\right) \cap\left(\tau_{M-l} \leq u\right)
$$

and so $B_{l} \in \mathscr{F}_{u}$.

Therefore, we have proved (3.3)

Q.E. D.

In the sequel we shall assume that the random variable $X_{1}^{(M)}(0)$ is independent of the standard Poissson process $N(*)$. As an application of LEMMA 3.1, we shall show

THEOREM 3.1. For each fixed $M>0,\left(\Omega, \sigma\left(X_{1}(s) ; 0 \leq s \leq t\right), X_{1}^{(M)}(*), P\right)$ is a Markov process.

PROOF. We have only to prove that, for any fixed $s, t, 0 \leq s<t$, there exists a random variable $Y$, independent of $\sigma\left(X_{1}^{(M)}(\tau) ; 0 \leq \tau \leq s\right)$, such that $X_{1}^{(M)}(t)$ can be represented as a Borel function of $X_{1}^{(M)}(s)$ and $Y$ (ITO [1]). We can rewrite equation (2.1) into

$$
X_{1}^{(M)}(t)=X_{1}^{(M)}(s)+N_{1}\left(\frac{\lambda}{M} \int_{s}^{t} X_{1}^{(M)}(\tau) d \tau\right),
$$

where $N_{1}(*)$ is a stochastic process defined by

$$
N_{1}(u) \equiv N\left(T^{(M)}(s)+u\right)-N\left(T^{(M)}(s)\right) \quad(u \geq 0) .
$$

We can see from the method of the construction of the unique solution of equation (2.1) that, for any $\tau>0$, there exists a Borel function $F_{\tau}$ from $\boldsymbol{R} \times \boldsymbol{R}^{[0, \infty)}$ into $\boldsymbol{R}$ such that

$$
X_{1}^{(M)}(\tau)=F_{\tau}\left(X_{1}^{(M)}(0),(N(u) ; u \geq 0)\right) .
$$

By noting equation (3.5), we find from the uniqueness of solution of equation (2.1) that

$$
X_{1}^{(M)}(t)=F_{t-s}\left(X_{1}^{(M)}(s),\left(N_{1}(u) ; u \geq 0\right)\right) .
$$

Next we shall show

(3. 8) $\quad N_{1}(*)$ is independent of $\sigma\left(X_{1}(\tau) ; 0 \leq \tau \leq s\right)$.

For this proof, we have only to prove that, for any $0 \leq \tau_{1}<\tau_{2}<\cdots<\tau_{p} \leq s$, $0 \leq u_{1}<u_{2}<\cdots<u_{q}$ and any smooth functions $G, H$,

$$
\begin{aligned}
& E\left(G\left(X_{1}^{(M)}\left(\tau_{1}\right), \cdots, X_{1}^{(M)}\left(\tau_{p}\right)\right) H\left(N_{1}\left(u_{1}\right), \cdots, N_{1}\left(u_{q}\right)\right)\right) \\
& \quad=E\left(G\left(X_{1}^{(M)}\left(\tau_{1}\right), \cdots, X_{1}^{(M)}\left(\tau_{p}\right)\right)\right) E\left(H\left(N_{1}\left(u_{1}\right), \cdots, N_{1}\left(u_{q}\right)\right)\right) .
\end{aligned}
$$

We approximate $T^{(M)}\left(\tau_{l}\right), T^{(M)}(s)$ by the random times $T_{n}^{(M)}\left(\tau_{l}\right), T_{n}^{(M)}(s)$ with discrete values such that 


$$
\begin{cases}T_{n}^{(M)}\left(\tau_{l}\right) \equiv \frac{k}{2^{n}} & \text { if } \frac{k-1}{2^{n}} \leq \tau_{l}<\frac{k}{2^{n}} \\ T_{n}^{(M)}(s) \equiv \frac{k}{2^{n}} & \text { if } \frac{k-1}{2^{n}} \leq s<\frac{k}{2^{n}}\end{cases}
$$

Then we have

$$
\begin{aligned}
& E\left\{G\left(X_{1}^{(M)}\left(\tau_{1}\right), \cdots, X_{1}^{(M)}\left(\tau_{p}\right)\right) H\left(N_{1}\left(u_{1}\right), \cdots, N_{1}\left(u_{q}\right)\right)\right\} \\
& =\lim _{n \rightarrow \infty} E\left\{G\left(X_{1}^{(M)}(0)+N\left(T_{n}^{(M)}\left(\tau_{1}\right)\right), \cdots, X_{1}^{(M)}(0)+N\left(T_{n}^{(M)}\left(\tau_{p}\right)\right)\right) \times\right. \\
& \left.\quad \times H\left(N\left(T_{n}^{(M)}(s)+u_{1}\right)-N\left(T_{n}^{(M)}(s)\right), \cdots, N\left(T_{n}^{(M)}(s)+u_{q}\right)-N\left(T_{n}^{(M)}(s)\right)\right)\right\} .
\end{aligned}
$$

For each $n$,

$$
\begin{gathered}
E\left\{G\left(X_{1}^{(M)}(0)+N\left(T_{n}^{(M)}\left(\tau_{l}\right)\right), \cdots, X_{1}^{(M)}(0)+N\left(T_{n}^{(M)}\left(\tau_{p}\right)\right)\right) \times\right. \\
\left.\times H\left(N\left(T_{n}^{(M)}(s)+u_{1}\right)-N\left(T_{n}^{(M)}(s)\right), \cdots, N\left(T_{n}^{(M)}(s)+u_{q}\right)-N\left(T_{n}^{(M)}(s)\right)\right)\right\} \\
=\sum_{1 \leq k_{1} \leq k_{2} \leq \cdots \leq k_{p} \leq k}^{\infty} E\left\{G\left(X_{1}^{(M)}(0)+N\left(T_{n}^{(M)}\left(\tau_{1}\right)\right), \cdots, X_{1}^{(M)}(0)+N\left(T_{n}^{(M)}\left(\tau_{p}\right)\right)\right) \times\right. \\
\times H\left(N\left(T_{n}^{(M)}(s)+u_{1}\right)-N\left(T_{n}^{(M)}(s)\right), \cdots, N\left(T_{n}^{(M)}(s)+u_{q}\right)-N\left(T_{n}^{(M)}(s)\right)\right) \\
\left.\quad ; \bigcap_{i=1}^{p}\left(\frac{k_{i}-1}{2^{n}} \leq T^{(M)}\left(\tau_{i}\right)<\frac{k_{i}}{2^{n}}\right) \cap\left(\frac{k-1}{2^{n}} \leq T^{(M)}(s)<\frac{k}{2^{n}}\right)\right\} \\
=\sum_{1 \leq k_{1} \leq k_{2} \leq \cdots \leq k_{p} \leq k}^{\infty} E\left\{G\left(X_{1}^{(M)}(0)+N\left(\frac{k_{1}}{2^{n}}\right), \cdots, X_{1}^{(M)}(0)+N\left(\frac{k_{p}}{2^{n}}\right)\right) \times\right. \\
\quad \times H\left(N\left(\frac{k}{2^{n}}+u_{1}\right)-N\left(\frac{k}{2^{n}}\right), \cdots, N\left(\frac{k}{2^{n}}+u_{q}\right)-N\left(\frac{k}{2^{n}}\right)\right) \\
\left.; \bigcap_{i=1}^{p}\left(\frac{k_{i}-1}{2^{n}} \leq T^{(M)}\left(\tau_{i}\right)<\frac{k_{i}}{2^{n}}\right) \cap\left(\frac{k-1}{2^{n}} \leq T^{(M)}(s)<\frac{k}{2^{n}}\right)\right\} .
\end{gathered}
$$

By LEMMA 3.1, the events $\bigcap_{i=1}^{p}\left(\frac{k_{i}-1}{2^{n}} \leq T^{(M)}\left(\tau_{i}\right)<\frac{k_{i}}{2^{n}}\right)$ and $\left(\frac{k-1}{2^{n}} \leq T^{(M)}(s)<\frac{k}{2^{n}}\right)$ belong to the $\sigma$-field $\mathscr{F}_{\frac{k}{2^{n}}}^{(M)}$. Since $H\left(N\left(\frac{k}{2^{n}}+u_{1}\right)-N\left(\frac{k}{2^{n}}\right), \cdots, N\left(\frac{k}{2^{n}}+u_{q}\right)\right.$ $\left.-N\left(\frac{k}{2^{n}}\right)\right)$ is independent of $\mathscr{F}_{\frac{k}{2^{n}}}^{(M)}$, we find from the temporal homogenuity of the standard Poisson process $N(*)$ that

$$
\begin{gathered}
E\left\{G \left(X_{1}^{(M)}(0)+N\left(T_{n}^{(M)}\left(\tau_{1}\right), \cdots, X_{1}^{(M)}(0)+N\left(T_{n}^{(M)}\left(\tau_{p}\right)\right)\right) \times\right.\right. \\
\left.\times H\left(N\left(T_{n}^{(M)}(s)+u_{1}\right)-N\left(T_{n}^{(M)}(s)\right), \cdots, N\left(T_{n}^{(M)}(s)+u_{q}\right)-N\left(T_{n}^{(M)}(s)\right)\right)\right\} \\
=\sum_{1 \leq k_{1} \leq k_{2} \leq \cdots \leq k_{p} \leq k}^{\infty} E\left\{G\left(X_{1}^{(M)}(0)+N\left(T_{n}^{(M)}\left(\tau_{1}\right)\right), \cdots, X_{1}^{(M)}(0)+N\left(T_{n}^{(M)}\left(\tau_{p}\right)\right)\right)\right. \\
\left.; \bigcap_{i=1}^{p}\left(\frac{k_{i}-1}{2^{n}} \leq T^{(M)}\left(\tau_{i}\right)<\frac{k_{i}}{2^{n}}\right) \cap\left(\frac{k-1}{2^{n}} \leq T^{(M)}(s)<\frac{k}{2^{n}}\right)\right\} \times \\
\quad \times E\left\{H\left(N\left(T_{n}^{(M)}(s)+u_{1}\right)-N\left(T_{n}^{(M)}(s)\right), \cdots, N\left(T_{n}^{(M)}(s)+u_{q}\right)-N\left(T_{n}^{(M)}(s)\right)\right)\right\} \\
=\sum_{k \geq 1}^{\infty} E\left\{G\left(X_{1}^{(M)}(0)+N\left(T_{n}^{(M)}\left(\tau_{1}\right)\right), \cdots, X_{1}^{(M)}(0)+N\left(T_{n}^{(M)}\left(\tau_{p}\right)\right)\right)\right.
\end{gathered}
$$




$$
\begin{gathered}
\left.; \frac{k-1}{2^{n}} \leq T^{(M)}(s)<\frac{k}{2^{n}}\right\} \times E\left\{H\left(N\left(\frac{k}{2^{n}}+u_{1}\right)-N\left(\frac{k}{2^{n}}\right)\right), \cdots,\right. \\
\left.\left.N\left(\frac{k}{2^{n}}+u_{q}\right)-N\left(\frac{k}{2^{n}}\right)\right)\right\} \\
=\sum_{k \geq 1}^{\infty} E\left\{G\left(X_{1}^{(M)}(0)+N\left(T_{n}^{(M)}\left(\tau_{1}\right)\right), \cdots, X_{1}^{(M)}(0)+N\left(T_{n}^{(M)}\left(\tau_{p}\right)\right)\right)\right. \\
\left.; \frac{k-1}{2^{n}} \leq T^{(M)}(s)<\frac{k}{2^{n}}\right\} \times E\left\{H\left(N\left(u_{1}\right)-N(0), \cdots, N\left(u_{q}\right)-N(0)\right)\right\} \\
=E\left\{G\left(X_{1}^{(M)}(0)+N\left(T_{n}^{(M)}\left(\tau_{1}\right)\right), \cdots, X_{1}^{(M)}(0)+N\left(T_{n}^{(M)}\left(\tau_{p}\right)\right)\right)\right\} \times \\
\quad \times E\left\{H\left(N\left(u_{1}\right)-N(0), \cdots, N\left(u_{q}\right)-N(0)\right)\right\} .
\end{gathered}
$$

Letting $n$ tend to $\infty$, we have

$$
\begin{aligned}
& E\left\{G\left(X_{1}^{(M)}\left(\tau_{1}\right), \cdots, X_{1}^{(M)}\left(\tau_{p}\right)\right) H\left(N_{1}\left(u_{1}\right), \cdots, N_{1}\left(u_{q}\right)\right)\right\} \\
& \left.\quad=E\left\{G\left(X_{1}^{(M)}\left(\tau_{1}\right), \cdots, X_{1}^{(M)}\left(\tau_{p}\right)\right)\right\} \cdot E\left\{H\left(N\left(u_{1}\right)-N(0)\right), \cdots, N\left(u_{q}\right)-N(0)\right)\right\} .
\end{aligned}
$$

By the same procedure, we can see that

$$
E\left\{H\left(N_{1}\left(u_{1}\right), \cdots, N_{1}\left(u_{q}\right)\right)\right\}=E\left\{H\left(N\left(u_{1}\right)-N(0), \cdots, N\left(u_{q}\right)-N(0)\right)\right\} .
$$

Therefore we find that (3.9) and so (3.8) holds. By combining (3.8) with (3.7), we have completed the proof of THEOREM 3.1.

Set

(3. 10) $\quad \mathscr{M}^{(M)}(t) \equiv N\left(T^{(M)}(t)\right)-T^{(M)}(t)$

and

(3. 11) $\quad \widetilde{\mathscr{F}}_{t}^{(M)} \equiv \mathscr{F}_{T^{(M)}(t)}^{(M)}$.

It then follows from equation (2.1) that

$$
\begin{aligned}
& X_{1}^{(M)}(t)=X_{1}^{(M)}(0)+\mathscr{M}^{(M)}(t)+T^{(M)}(t), \\
& X_{2}^{(M)}(t)=X_{2}^{(M)}(0)-\mathscr{M}^{(M)}(t)-T^{(M)}(t) .
\end{aligned}
$$

THEOREM 3.2. The stochastic processes $X_{j}^{(M)}(*)$ are $\left(\tilde{F}_{t}^{(M)} ; t \geq 0\right)$ semimartingales such that

(i ) $\mathscr{M}^{(M)}(*)$ is a square-integrable and $\left(\tilde{F}_{t}^{(M)} ; t \geq 0\right)$-martingale,

(ii) $T^{(M)}(*)$ is a continuous, increasing and $\left(\widetilde{\mathscr{F}}_{t}^{(M)} ; t \geqq 0\right)$-adapted process,

(iii) $\left\langle\mathscr{M}^{(M)}\right\rangle_{t}=T^{(M)}(t) \quad(t \geq 0)$.

ProOF. Put

(3. 14) $\tilde{N}(t) \equiv N(t)-t$,

(3. 15) $A(t) \equiv t$. 
Then we have

$$
N(t)=\tilde{N}(t)+A(t)
$$

It is well known that (3.16) is the Doob-Meyer decomposition, that is, $\widetilde{N}(*)$ is a square integrable martingale part and $A(*)$ is a natural increasing process part of the standard Poisson process $N(*)$. Moreover we know that the quadratic variational process of $\widetilde{N}(*)$ equals $A(*)$ :

$$
\langle\tilde{N}\rangle_{t}=A(t)
$$

Since $T^{(M)}(*)$ is a bounded continuous $\left(\mathscr{F}_{t}^{(M)} ; t \geq 0\right)$-stopping time, by virtue of LEMMA 3.1, we can apply the optional sampling theorem due to Doob to (3.16) and (3.17) to conclude that THEOREM 3.2 holds. Q.E.D.

We shall explain why the relation (iii) in THEOREM 3.2 can be regarded as a kind of the fluctuation-dissipation theorem in statistical physics (KUBO [6], OKABE [11-16]). As stated in OKABE $[13,14]$, the heart in the fluctuation-dissipation theorem lies in a philosophical understanding that any phenomena in a complex system, its equation of motion describing the time evolution, under certain stationary situation, can be separated into a random chaotic part (fluctuating term) and a dynamical calm part (dissipative term) and certain relation holds between both parts. The stochastic process $X_{1}^{(M)}(*)$ does not have any stationary property, but has a Markovian property. Moreover the decomposition (3.12) as the semimartingale can be regarded as the one into the fluctuating part and the dissipative part of the system $X_{1}^{(M)}(*)$, which are given as a martingale and a bounded variation process, respectively. The relation (iii) in THEO. REM 3.2 gives a relation between both parts. For this reason we shall call the relation (iii) in THEOREM 3.2 the fluctuation-dissipation theorem. The situation holds also for the Markov processes $X_{2}^{(M)}(*)$ and $N(*)$. In the future we shall investigate what a qualitative property characterizes such a fluctuation-dissipation theorem.

\section{§ 4. The law of large numbers}

Let $u_{1}=u_{1}(t)$ and $u_{2}=u_{2}(t)(t \in[0, \infty))$ be the unique solution of the following Lotka-Volterra equation

$$
\left\{\begin{array}{l}
\frac{d u_{1}(t)}{d t}=\lambda u_{1}(t) u_{2}(t), \\
\frac{d u_{2}(t)}{d t}=-\lambda u_{1}(t) u_{2}(t) .
\end{array}\right.
$$

The purpose in this section is to discuss the convergence of the ratios 
$\frac{X_{1}^{(M)}(t)}{M}$ and $\frac{X_{2}^{(M)}(t)}{M}$ to $u_{1}(t)$ and $u_{2}(t)$, respectively, as the total number $M$ of particles tends to $\infty$.

By the same method as in the queuing model of LIPTSER-SHIRYAEV [8], we can show the following lemma.

LEMMA 4.1. Let $w=w(t)(t \in[0, \infty))$ be the unique solution of the ordinary differential equation

$$
\frac{d w(t)}{d t}=f(w(t))
$$

satisfying the property with inf $f_{s s t} w(s)>0$ for any $t>0$. Here $f=f(x)$ is a non-negative function on $[0, \infty)$ with local Lipschitz condition.

For each $M>0$, let $Y^{(M)}(*)$ be an $\left(\mathscr{H}_{t}^{(M)} ; t \geq 0\right)$-semimartingale on a probability space such that

(i ) $Y^{(M)}(t)=Y^{(M)}(0)+\mathscr{M}^{(M)}(t)+\mathscr{A}^{(M)}(t)$,

(ii) $\mathscr{M}^{(M)}(*)$ is a square integrable and $\left(\mathscr{H}_{t}^{(M)} ; t \geq 0\right)$-martingale,

(iii) $\mathscr{A}^{(M)}(*)$ is a continuous, increasing and $\left(\mathscr{H}_{t}^{(M)} ; t \geq 0\right)$-adapted process,

(iv) $\mathscr{A}^{(M)}(t)=M \int_{0}^{t} f\left(\frac{Y^{(M)}(s)}{M}\right) d s$,

( v ) $\left\langle\mathscr{M}^{(M)}\right\rangle_{t}=\mathscr{A}^{(M)}(t)$.

Moreover we assume that

$$
\lim _{M \rightarrow \infty} \frac{Y^{(M)}(0)}{M}=w(0) \text { in probability. }
$$

Then for any $t>0$,

$$
\lim _{M \rightarrow \infty} \frac{Y^{(M)}(t)}{M}=w(t) \text { in probability. }
$$

By applying LEMMA 4.1 to THEOREM 3.1, we have

THEOREM 4.1 (The weak law of large numbers). We assume

$$
\left\{\begin{array}{l}
\lim _{M \rightarrow \infty} \frac{X_{1}^{(M)}(0)}{M}=u_{1}(0) \text { in probability, } \\
0<u_{1}(0)<1 \text { and } u_{1}(0)+u_{2}(0)=1 .
\end{array}\right.
$$

Then for any $t>0$,

$$
\begin{aligned}
& \lim _{M \rightarrow \infty} \frac{X_{1}^{(M)}(t)}{M}=u_{1}(t) \text { in probability, } \\
& \lim _{M \rightarrow \infty} \frac{X_{2}^{(M)}(t)}{M}=u_{2}(t) \text { in probability. }
\end{aligned}
$$


As a strong assertion of THEOREM 4.1, we shall show

THEOREM 4.2 (The strong law of large numbers). We assume

$$
\left\{\begin{array}{l}
\lim _{M \rightarrow \infty} \frac{X_{1}^{(M)}(0)}{M}=u_{1}(0) \text { a.s. } \\
0<u_{1}(0)<1 \text { and } u_{1}(0)+u_{2}(0)=1 .
\end{array}\right.
$$

Then for any $t>0$,

$$
\begin{aligned}
& \lim _{M \rightarrow \infty} \frac{X_{1}^{(M)}(t)}{M}=u_{1}(t) \quad \text { a.s. } \\
& \lim _{M \rightarrow \infty} \frac{X_{2}^{(M)}(t)}{M}=u_{2}(t) \quad \text { a.s. }
\end{aligned}
$$

PROOF. We rewrite (2.2) in an integral form

$$
\frac{X_{1}^{(M)}(t)}{M}=\frac{X_{1}^{(M)}(0)}{M}+\int_{0}^{\frac{M-X_{1}^{(M)}(0)}{M}} \varphi_{M}(p) d p
$$

where the function $\varphi_{M}(*)$ is defined on $[0, \infty)$ by

$$
\varphi_{M}(p) \equiv \chi_{\left[\sigma_{k}^{(M)}, \infty\right)}(t) \text { for } \frac{k-1}{M} \leq p<\frac{k}{M}
$$

We fix any element $\omega \in \Omega$ such that

$$
\lim _{M \rightarrow \infty} \frac{X_{1}^{(M)}(0)(\omega)}{M}=u_{1}(0),
$$

$$
\begin{aligned}
& \lim _{M \rightarrow \infty} \frac{1}{M} \sum_{i=1}^{M}\left(\tau_{i}(\omega)-\tau_{i-1}(\omega)\right)=1, \\
& \lim _{M \rightarrow \infty} \frac{1}{M} \sum_{i=1}^{M}\left(\tau_{i}(\omega)-\tau_{i-1}(\omega)\right)^{2}=2 .
\end{aligned}
$$

From our assumption and the fact that the strong law of large numbers holds for independent and identically distributed random variables $\tau_{i}-\tau_{i-1}$, $i=1,2, \cdots$, we note that the set of elements $\omega \in \Omega$ satisfying (4.5)-(4.7) has the probability one.

[Step 1] We claim that

$$
\frac{X_{1}^{(M)}(t)}{M}=\frac{X_{1}^{(M)}(0)}{M}+\int_{0}^{1-u_{1}(0)} \varphi_{M}(p) d p+O(1)(M \rightarrow \infty) .
$$

By (4.3), we have 


$$
\frac{X_{1}^{(M)}(t)}{M}=\left\{\begin{aligned}
\frac{X_{1}^{(M)}(0)}{M}+\int_{0}^{1-u_{1}(0)} \varphi_{M}(p) d p+ & \int_{1-u_{1}(0)}^{\frac{M-X^{(M)}(0)}{M}} \varphi_{M}(p) d p \\
& \text { for } 1-u_{1}(0) \leq \frac{M-X_{1}^{(M)}(0)}{M} \\
\frac{X_{1}^{(M)}(0)}{M}+\int_{0}^{1-u_{1}(0)} \varphi_{M}(p) d p-\int_{\frac{M-X^{M}(0)}{M}}^{1-u_{1}(0)} \varphi_{M}(p) d p & \text { for } \frac{M-X_{1}^{(M)}(0)}{M} \leq 1-u_{1}(0)
\end{aligned}\right.
$$

Since $0 \leq \varphi_{M}(p) \leq 1$.

$$
\frac{X_{1}^{(M)}(t)}{M}=\frac{X_{1}^{(M)}(0)}{M}+\int_{0}^{1-u_{1}(0)} \varphi_{M}(p) d p+O\left(\left|u_{1}(0)-\frac{X_{1}^{(M)}(0)}{M}\right|\right)(M \rightarrow \infty) .
$$

Hence Step 1 holds.

For the proof of THEOREM 4.2, we shall investigate the convergence of the integrand $\varphi_{M}(*)$ in (4.4) and so $\sigma_{k}^{(M)}$ in (2.4).

Let $s \in[0, \infty)$ be any real number such that

$$
0 \leq s<1-u_{1}(0)
$$

We take for each $M>0$ an integer $k_{M}$ such that $\frac{k_{M}-1}{M} \leq s<\frac{k_{M}}{M}$. It is to be noted that $\frac{k_{M}}{M}$ converges to $s$ as $M$ tends to $\infty$. We decompose $\sigma_{k_{M}}^{(M)}$ into

$$
\sigma_{k_{M}}^{(M)}=S_{1}+S_{2}+S_{3}
$$

where

$$
\begin{gathered}
S_{1}=\frac{1}{M \lambda} \sum_{i=1}^{\infty}\left(\chi_{\left\{i \leq k_{M}\right\}}-\chi_{\{i \leq[M s]\}}\right) \frac{\tau_{i}-\tau_{i-1}}{\frac{X_{1}^{(M)}(0)+i-1}{M}\left(1-\frac{X_{1}^{(M)}(0)+i-1}{M}\right)} \\
S_{2}=\frac{1}{M \lambda} \sum_{i=1}^{\infty} \chi_{\{i \leq[M s]\}}\left\{\frac{\tau_{i}-\tau_{i-1}}{\frac{X_{1}^{(M)}(0)+i-1}{M}\left(1-\frac{X_{1}^{(M)}(0)+i-1}{M}\right)}\right. \\
\left.-\frac{\tau_{i}-\tau_{i-1}}{\left(u_{1}(0)+\frac{i-1}{M}\right)\left(1-u_{1}(0)-\frac{i-1}{M}\right)}\right\} \\
S_{3}=\frac{1}{M \lambda} \sum_{i=1}^{\infty} \chi_{\{i \leq[M s]\}} \frac{\tau_{i}-\tau_{i-1}}{\left(u_{1}(0)+\frac{i-1}{M}\right)\left(1-u_{1}(0)-\frac{i-1}{M}\right)}
\end{gathered}
$$

[Step 2] We shall prove that $\lim _{M \rightarrow \infty} S_{1}=0$.

By (4.7), there exists a positive constant $C_{1}$ such that, for any $M>0$, 


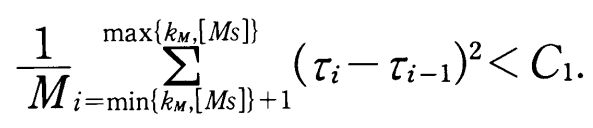

Moreover it follows from (4.5) that there exists a positive integer $M_{0}$ such that, for any $M \geq M_{0}$,

$$
\frac{u_{1}(0)}{2}<\frac{X_{1}^{(M)}(0)}{M}<u_{1}(0)+\frac{1-u_{1}(0)-s}{2} .
$$

Hence

$$
\begin{aligned}
& \left.\left|S_{1}\right| \leq\left\{\frac{1}{M_{i}} \sum_{i=\min \left\{\left\{k_{M}[M S\right.\right.}^{\max \left\{k_{M}\left[\left[M_{s}\right]\right\}+1\right.}\right\}\left(\tau_{i}-\tau_{i-1}\right)^{2}\right\}^{1 / 2} \times \\
& \times\left\{\frac{1}{M \lambda^{2}}{ }_{i=\min \left\{k_{M},[M s]\right\}+1}^{\max \left\{k_{M}[M s]\right\}} \frac{1}{\left(\frac{X_{1}^{(M)}(0)+i-1}{M} \frac{M-\left(X_{1}^{(M)}(0)+i-1\right.}{M}\right)^{2}}\right\}^{1 / 2} \\
& \leq\left\{C_{1}\right\}^{1 / 2}\left\{\frac{1}{M \lambda^{2}} \sum_{i=\min \left\{\left\langle k_{m}[M s]\right\}+1\right.}^{\max \left\{k_{m}[M s]\right\}}\left(\frac{1}{\frac{u_{1}(0)}{2} \frac{1-u_{1}(0)-s}{2}}\right)^{2}\right\}^{1 / 2} \\
& \leq\left\{C_{1}\right\}^{1 / 2}\left\{\frac{\left|k_{M}-M s\right|}{M \lambda^{2}}\left(\frac{1}{\frac{u_{1}(0)}{2} \frac{1-u_{1}(0)-s}{2}}\right)^{2}\right\}^{1 / 2} \text {. }
\end{aligned}
$$

Therefore we find that Step 2 holds.

[Step 3] We shall show that $\lim _{M \rightarrow \infty} S_{2}=0$.

By (4.7), there exists a positive constant $C_{2}$ such that, for any $M>0$,

$$
\frac{1}{M} \sum_{i=1}^{[M s]}\left(\tau_{i}-\tau_{i-1}\right)^{2}<C_{2}
$$

Hence, by using (4.9) in the proof of Step 2, for any $M \geq M_{0}$, we have the estimate :

$$
\begin{aligned}
&\left|S_{2}\right| \leq\left\{\frac{1}{M} \sum_{i=1}^{[M S]}\left(\tau_{i}-\tau_{i-1}\right)^{2}\right\}^{1 / 2} \times \\
& \times\left\{\frac{1}{M \lambda^{2}} \sum_{i=1}^{[M S !}\left(\frac{\left(1-\frac{X_{1}^{(M)}(0)}{M}-\frac{i-1}{M}\right)-\left(u_{1}(0)+\frac{i-1}{M}\right)}{\left(\frac{X_{1}^{(M)}(0)}{M}+\frac{i-1}{M}\right)\left(1-\frac{X_{1}^{(M)}(0)}{M}-\frac{i-1}{M}\right)\left(u_{1}(0)+\frac{i-1}{M}\right)\left(1-u_{1}(0)-\frac{i-1}{M}\right)}\right)^{2}\right\}^{1 / 2} \times \\
& \times\left|\frac{X_{1}^{(M)}(0)}{M}-u_{1}(0)\right| \\
& \leq\left\{C_{2}\right\}^{1 / 2}\left\{\frac { s } { \lambda ^ { 2 } } \left(\frac{1}{\frac{u_{1}(0)}{2} u_{1}(0)\left(1-u_{1}(0)-s\right)}+\right.\right.
\end{aligned}
$$




$$
\begin{aligned}
& \left.\left.+\frac{1}{\frac{u_{1}(0)}{2} \frac{1-u_{1}(0)-s}{2}\left(1-u_{1}(0)-s\right)}\right)^{2}\right\}^{1 / 2} \times \\
& \times\left|\frac{X_{1}^{(M)}(0)}{M}-u_{1}(0)\right| .
\end{aligned}
$$

Therefore we find from (4.5) that Step 3 holds.

[Step 4] We shall prove that

$$
\lim _{M \rightarrow \infty} S_{3}=\frac{1}{\lambda} \log \frac{\left(u_{1}(0)+s\right)\left(1-u_{1}(0)\right)}{u_{1}(0)\left(1-u_{1}(0)-s\right)} .
$$

By defining a sequence of Borel measures $\left\{\mu_{M} ; M=1,2, \cdots\right\}$ on $[0, s]$ such that

$$
\mu_{M}\left(\left\{\frac{i-1}{M}\right\}\right) \equiv \frac{1}{M}\left(\tau_{i}-\tau_{i-1}\right) \quad(1 \leq i \leq[M s]) .
$$

We can rewrite $S_{3}$ into

$$
S_{3}=\int_{0}^{s} g(p) d \mu_{M}(d p)
$$

where $g$ is a continuous function on $[0, s]$ such that

$$
g(p)=\frac{1}{\lambda\left(u_{1}(0)+p\right)\left(1-u_{1}(0)-p\right)}
$$

Since it can be seen that the weak law of large numbers (4.6) implies that

$$
w-\lim _{M \rightarrow \infty} \mu_{M}=\text { the Lebesgue measure on }[0, s],
$$

we find that

$$
\lim _{M \rightarrow \infty} S_{3}=\int_{0}^{s} g(p) d p
$$

A direct calculation gives us that Step 4 holds.

[Step 5] It follows from Step 1-Step 4 that the following limit exists :

$$
\sigma(s) \equiv \lim _{M \rightarrow \infty} \sigma_{k_{M}}^{(M)}=\frac{1}{\lambda} \log \frac{\left(u_{1}(0)+s\right)\left(1-u_{1}(0)\right)}{u_{1}(0)\left(1-u_{1}(0)-s\right)}
$$

and so the $\operatorname{limit} \lim _{M \rightarrow \infty} \frac{X_{1}^{(M)}(t)}{M}$ exists and it is equal to 


$$
u_{1}(0)+\int_{0}^{1-u_{1}(0)} \chi_{[\sigma(s), \infty)}(t) d s=\frac{u_{1}(0) e^{\lambda t}}{u_{1}(0) e^{\lambda t}+1-u_{1}(0)}
$$

This is a logistic distribution, which coincides with the unique solution of Lotka-Volterra equation (4.1).

Consequently we have completed the proof of THEOREM 4.2. Q. E. D.

\section{$\S 5$. The central limit theorem}

For each $M>0$, we define a stochastic process $Y_{1}^{(M)}(*)$ by

$$
Y_{1}^{(M)}(t) \equiv \sqrt{M}\left(\frac{X_{1}^{(M)}(t)}{M}-u_{1}(t)\right) .
$$

By the same method as in the queuing model of LIPTSER-SHIRYAEV [8], we can show the following central limit theorem.

THEOREM 5.1. Let the sequence of random variables $\left(Y_{1}^{(M)}(0)\right)_{M \geq 1}$ converges weakly to a probability distribution $G$ defined on $\boldsymbol{R}$.

Then the sequence of the stochastic processes $\left(Y_{1}^{(M)}(t) ; t \geq 0\right)_{M \geq 1}$ converges weakly to a one-dimensional Gaussian diffusion process $\left(Y_{1}(t) ; t \geq 0\right)$ whose time evolution is governed by the following stochastic differential equation

$$
d Y_{1}(t)=\lambda\left(u_{1}(t)+\left(u_{2}(t)-u_{1}(t)\right) Y_{1}(t)\right) d t+\sqrt{\lambda u_{1}(t)\left(1-u_{1}(t)\right)} d B(t),
$$

where $B(*)$ is a one-dimensional Brownian motion.

\section{References}

[1] ITO, K., Probability Theory, Iwanami Shoten, Tokyo, 1953 (in Japanese).

[2] ITOH, Y., On a ruin problem with interaction, Ann. Inst. Statist. Math., 25 (1973), 854 -858 .

[3] , Random collision models in oriented graphs, J. Appl. Prob., 16 (1979), 36 -44 .

[4] - Representation for Wright model in population genetics, Research Memorandum, No. 201 (1981), 1-9, The Institute of Statistical Mathematics, Tokyo.

[5] KAC. M., Probability and related topics in physical sciences, Interscience Publisher, London, 1959.

[6] KUBO, R., Statistical mechanical theory of irreversible processes I, general theory and simple applications to magnetic and conduction problems, J. Phys. Soc. Japan, 12 (1957), 570-586.

[ 7 ] Kogan, Ya. A., R. Sh. LIPTSER, and A. V. SMORODINSKI, Gaussian diffusion approximation of closed Markov models of computer networks, Prob. Inf. Transmission, 22 (1986), 38-51.

[8] Liptser, R. Sh., and A. N. Shiryaev, Martingale Theory, Kluwer Academic Publishers, Dordrecht, 1989.

[ 9 ] LotKA, A. J., Elements of physical biology, Williams \& Wilkins, Baltimore, 1925. 
[10] Moran, P. A. P., Random processes in genetics, Proc. Camb. Phil. Soc., 54 (1958), 6071.

[11] OKABE, Y., On KMO-Langevin equations for stationary Gaussiant processes with T-positivity, J. Fac. Sci. Univ. Tokyo, Sect. IA Math., 33 (1986), 1-56.

[12] On the theory of Brownian motion with the Alder-Wainwright effect, J. Stat. Phys., 45 (1986), 953-981.

[13] Langevin equation and causal analysis, S Japanese).

[14] Langevin equation and causal analysis, to appear in Sugaku Expositions, Amer. Math. Soc.

[15] OKABE, Y. and Y. NAKANO, The theory of $\mathrm{KM}_{2} \mathrm{O}$-Langevin equations and its applications to data analysis (I) : Stationay analysis, Hokkaido Math. J., 20 (1991), 4590.

[16] OKABE, Y. and A. InOUE, The theory of $\mathrm{KM}_{2} \mathrm{O}$-Langevin equations and its applications to data analysis (II) : Causal analysis, Nagoya Math. J., 134 (1994), 1-28.

[17] OKABE, Y., H. MANO and Y. ITOH, Random collision model for interacting populations of two species and its strong law of large numbers, Research Memorandum, No. 484 (1993), 1-13, The Institute of Statistical Mathematics, Tokyo.

[18] - Paper-scissors-stone model for interacting population and its limit theorem, Research Memorandum, No. 485 (1993), 1-37, The Institute of Statistical Mathematics, Tokyo.

[19] Volterra, V., Leçons sur la theorie mathematique de la lutte pour la vie, Scientifique VII, Gauthier-Villars, Paris, 1931.

Department of Mathematics

Faculty of Science

Hokkaido University

Sapporo

060

Current Address :

Department of Mathematical Engineering and Information Physics

Faculty of Engineering

The University of Tokyo

Hongo, 113

Japan

The Institute of Statistical Mathematics

The Graduate University for Advanced Studies

Tokyo

106

The Institute of Statistical Mathematics

The Graduate University for Advanced Studies

Tokyo

106 